\title{
Extracellular vesicles and pasteurized cells derived from Akkermansia muciniphila protect against high-fat induced obesity in mice
}

Fatemeh Ashrafian 1,2, Shahrbanoo Keshavarz Azizi Raftar ${ }^{1}$, Arezou Lari ${ }^{3}$, Arefeh Shahryari' , Sara Abdollahiyan ${ }^{1,4}$, Hamid Reza Moradi ${ }^{5}$, Morteza Masoumi ${ }^{1,6}$, Mehdi Davari ${ }^{1,6}$, Shohreh khatami ${ }^{7}$, Mir Davood Omrani ${ }^{8}$, Farzam Vaziri ${ }^{1,6}$, Andrea Masotti $^{9}$ and Seyed Davar Siadat ${ }^{1,6^{*}}$ (1)

\begin{abstract}
Background: Several studies have shown that probiotics have beneficial effects on weight control and metabolic health. In addition to probiotics, recent studies have investigated the effects of paraprobiotics and postbiotics. Therefore, we evaluated the preventive effects of live and pasteurized Akkermansia muciniphila MucT (A. muciniphila) and its extracellular vesicles (EVs) on HFD-induced obesity.

Results: The results showed that body weight, metabolic tissues weight, food consumption, and plasma metabolic parameters were increased in the HFD group, whereas A. muciniphila preventive treatments inhibited these HFD. The effects of pasteurized A. muciniphila and its extracellular vesicles were more noticeable than its active form. The HFD led to an increase in the colonic, adipose tissue, and liver inflammations and increased the expression of genes involved in lipid metabolism and homeostasis. Nevertheless, these effects were inhibited in mice that were administered A. muciniphila and its EVs. The assessment of the gut microbiota revealed significant differences in the microbiota composition after feeding with HFD. However, all treatments restored the alterations in some bacterial genera and closely resemble the control group. Also, the correlation analysis indicated that some gut microbiota might be associated with obesity-related indices.
\end{abstract}

Conclusions: Pasteurized A. muciniphila and its EVs, as paraprobiotic and postbiotic agents, were found to play a key role in the regulation of metabolic functions to prevent obesity, probably by affecting the gut-adipose-liver axis.

Keywords: Akkermansia muciniphila, Extracellular vesicles, Obesity, Pasteurization

\footnotetext{
*Correspondence: d.siadat@gmail.com

${ }^{1}$ Microbiology Research Center (MRC), Pasteur Institute of Iran, Tehran,

Iran

Full list of author information is available at the end of the article
}

(c) The Author(s) 2021. Open Access This article is licensed under a Creative Commons Attribution 4.0 International License, which permits use, sharing, adaptation, distribution and reproduction in any medium or format, as long as you give appropriate credit to the original author(s) and the source, provide a link to the Creative Commons licence, and indicate if changes were made. The images or other third party material in this article are included in the article's Creative Commons licence, unless indicated otherwise in a credit line to the material. If material is not included in the article's Creative Commons licence and your intended use is not permitted by statutory regulation or exceeds the permitted use, you will need to obtain permission directly from the copyright holder. To view a copy of this licence, visit http://creativecommons.org/licenses/by/4.0/. The Creative Commons Public Domain Dedication waiver (http://creativeco mmons.org/publicdomain/zero/1.0/) applies to the data made available in this article, unless otherwise stated in a credit line to the data. 


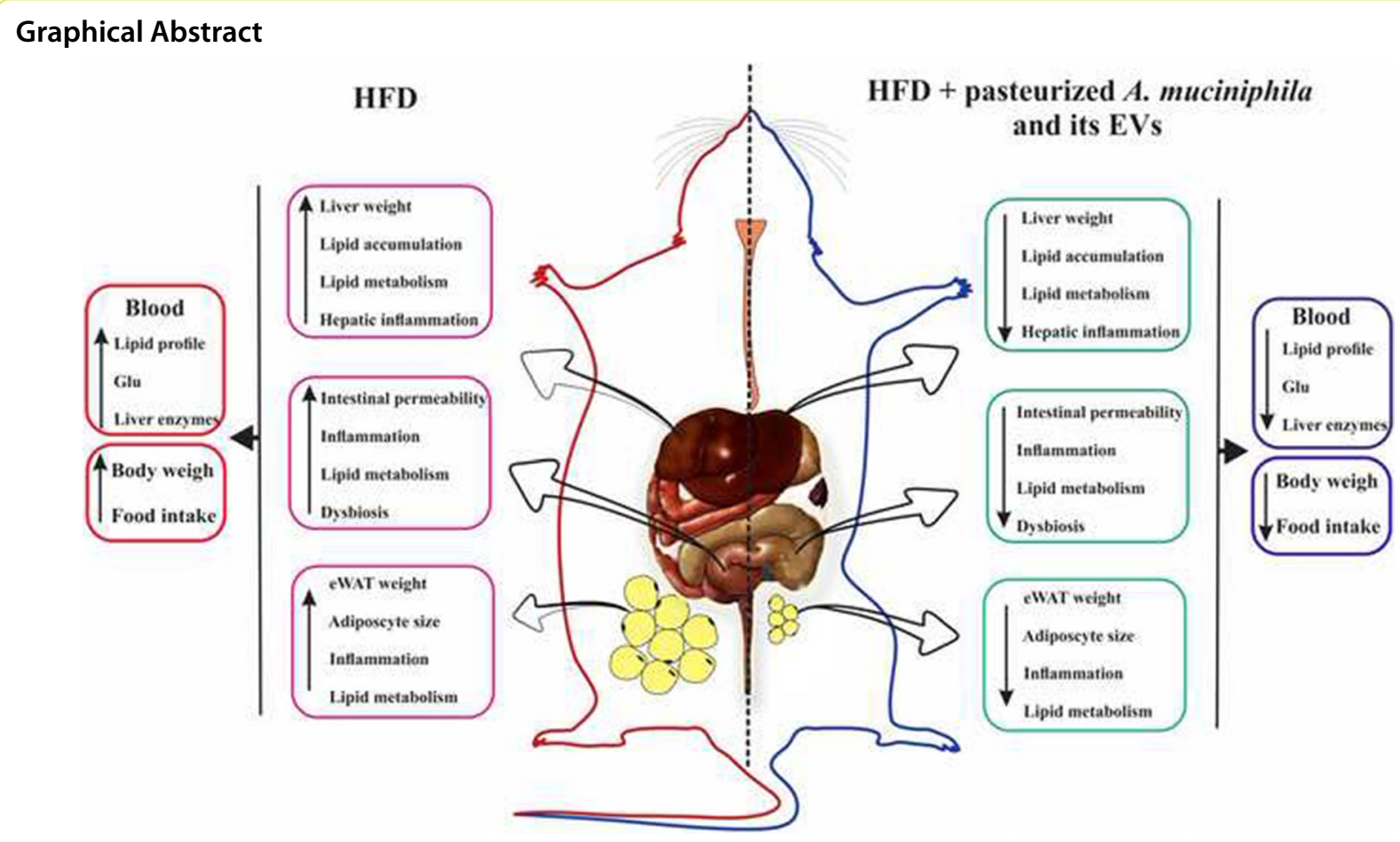

\section{Background}

The inter-organ communication is vital for the control and maintenance of energy, metabolism, and immune homeostasis. The phenotypic consequence of disruptions in this type of communication is obesity, which is one of the main public health and medical concerns worldwide $[1,2]$. So far, several therapeutic and preventive medications have been used for obesity management, which can increase energy expenditure and reduce fat absorption, energy intake, and appetite [3]; however, these medications have several side effects such as adverse cardiovascular, psychiatric, and gastrointestinal events [4].

Recently, probiotics have received considerable attention as beneficial agents for preventing obesity by manipulating the intestinal microbiota through improved the gut microbiota composition, the colonization resistance phenomenon, and production of various metabolites (e.g., short-chain fatty acids (SCFAs) and vitamins) [5-7]. The intestinal microbiota plays a major role in health and disease by modulating the immune response and host metabolism. Multiple studies have revealed that a high-fat diet (HFD) is correlated with the gut microbiota disturbances, intestinal barrier permeability, onset of intestinal, adipose, and hepatic inflammations, changes in lipid metabolism, and consequently, obesity-induced metabolic disorders $[8,9]$. HFD reduces the expression of tight junction proteins and increases intestinal permeability, leading to the transfer of bacterial fragments such as lipopolysaccharides (LPS) into the circulation and induction of metabolic endotoxemia. In addition, an increase in plasma LPS levels affects multiple organs and induces metabolic changes and systemic inflammation. These changes are associated with a significant reduction in the beneficial bacterial population and an increase in pathogenic bacteria in the gut [7]. However, the central mechanism and roles of inter-organ communication in the pathogenesis of obesity, have not been completely clarified.

Growing evidence shows that the gut microbiota plays a vital role in the management of the host metabolism through interplay between metabolic tissues [10]. Therefore, treatment of the gut microbiota by probiotics, postbiotics, and paraprobiotics is considered a potential strategy for the treatment and prevention of obesity $[11,12]$. It should be noted that paraprobiotics (cellular structural components) and postbiotics (metabolic products secreted by probiotics) are two forms of non-viable probiotics, when administrated in sufficient amounts, could have beneficial effects on host health [13]. Several strains of probiotics can reverse the HFD-induced adverse effects, including Akkermansia muciniphila (A. muciniphila), which is widely regarded as a next-generation probiotic [14-16]. Our previous 
research as well as many other studies have reported the beneficial effects of $A$. muciniphila on energy metabolism, fatty acid (FA) oxidation, inflammation, and gut integrity [15-20]. Considering the sensitivity of A. muciniphila to oxygen and its reduced efficacy during administration, recent studies have suggested the use of its inactive form or its derivatives rather than its live form $[15,16,21,22]$.

A recent study revealed that pasteurized A. muciniphila had more significant effects on metabolism as compared to its live form and could reduce the weight gain and increase the total energy expenditure [17]. The interaction of the intestinal microbiota with the host and regulation of multiple signaling pathways are triggered by the secretion of nano-sized extracellular vesicles (EVs), which can pass the mucus layers and transfer to peripheral tissues through circulation [22-24]. In this regard, many studies have reported the positive effects of the gut microbiota-derived EVs on the amelioration of several diseases [15, 21, 22]. However, further studies are needed to determine the precise mechanism of the microbiota EVs in the prevention of diseases.

Although several studies have been conducted on the effects of $A$. muciniphila cells, fragments and proteins in various diseases models, no systematic comparison of $A$. muciniphila-derived EVs, pasteurized and live cells have been reported. Therefore, in the present study, we aimed to use the well-defined model of diet-induced obesity to show the best effect of live and pasteurized A. muciniphila and its EVs to prevent weight gain, inflammation and lipid metabolism. We also aimed to compare the effects of live and pasteurized A. muciniphila and its EVs on the prevention of obesity.

\section{Results}

\section{Characterization of EVs}

The morphology and size of EVs derived from A. muciniphila were evaluated by SEM; the EVs were spherical shape and a range of $40-150 \mathrm{~nm}$ in size (Additional file 1: Fig. S1).

\section{A. muciniphila prevented increased food intake and plasma metabolic parameters in HFD-fed mice}

To assess the impact of live and pasteurized A. muciniphila and its EVs on preventing obesity, mice were fed an HFD for five weeks without or along with treatment.
The body weight of the HFD group was significantly enhanced than the ND group. All treatments prevented HFD-induced body weight gain, while pasteurized form significantly had a better effect than live form (Fig. 1b). Food intake also significantly increased in HFD mice than normal mice, while pasteurized A. muciniphila and its EVs significantly decreased food intake (Fig. 1c).

The glucose concentration in all treatments were significantly reduced relative to the HFD group, among which the HEV and HAm groups showed lower concentration. In comparison with ND mice, HFD increased plasma lipids profile level, however, all treatments showed preventive effects on HFD-induced hyperlipidemia. A significant reduction in TC level was observed in the HPAm group. All treatments significantly decreased TG and LDL levels as well as markedly increased the HDL concentration (Fig. 1d).

In the HPAm group, the greatest preventive effects on the plasma metabolic indicators of obesity were observed. The HFD markedly increased the plasma level of pro-inflammatory cytokines (i.e., TNF- $\alpha$ and IL-6) and decreased the level of IL-10 as an anti-inflammatory cytokine, compared to the ND-fed mice. However, all treatments could prevent the HFD-induced inflammation. Notably, live and pasteurized A. muciniphila induced the greatest effects on the reduction of pro-inflammatory cytokines, while the highest concentration of IL-10 was found in the HAm and HEV groups. Overall, the EVs and pasteurized A. muciniphila exerted more potential preventive effects on obesity, characterized by a decrease in body weight, blood biochemical parameters, and food intake.

\section{Expression of key genes involved in lipid metabolism and inflammation}

According to our meta-analysis, the expression of these genes in the liver was closely associated with the HFDinduced fatty liver, and they were differentially expressed $(\mathrm{P}<0.05)$. The heat-map plot revealed that lipid metabolism-associated genes (ppar- $\gamma$ and $l p l)$ and $\operatorname{tg} f-\beta$ were significantly enriched in the HFD group as compared to normal mice, while angptl4 and il-10 were lower in the HFD group, compared to normal mice (Fig. 2a). The principal component analysis (PCA) was also applied for the selected genes from all 22 ND and 52 HFD samples in four datasets. The results revealed that the ND group was

\footnotetext{
(See figure on next page.)

Fig. 1 The effects of alive, pasteurized A. muciniphila and its EVs on prevention of HFD-induced increase in body weight, food intake and plasma metabolic parameters in HFD-fed mice. a Experimental scheme, b Body weight, and c Average daily food intake in ND, HFD, and treatment groups. d The concentration levels of metabolic parameters e.g., Glucose, Triglyceride, Total cholesterol, LDL, HDL, and cytokines (i.e., TNF-a, IL-6, and IL-10) in the plasma of mice. Data are presented as the mean $\pm S D, N=7$ per group. ${ }^{\#} p<0.05 ; H F D$ vs. ND, ${ }^{a} p<0.05 ;{ }^{b} p<0.01$; treatment groups vs. HFD, and ${ }^{*} p<0.05 ;{ }^{* *} p<0.01$; among treatment groups were considered statistically significant, respectively. ND: normal diet + PBS, HFD: high fat diet + PBS, HAm: high fat diet + A. muciniphila $\left(10^{9} \mathrm{CFU}\right)$, HPAm: high fat diet + pasteurized A. muciniphila ( $\left.10^{9} \mathrm{CFU}\right)$, and HEV: high fat diet + EVs (10 $\mu \mathrm{g}$ protein)
} 


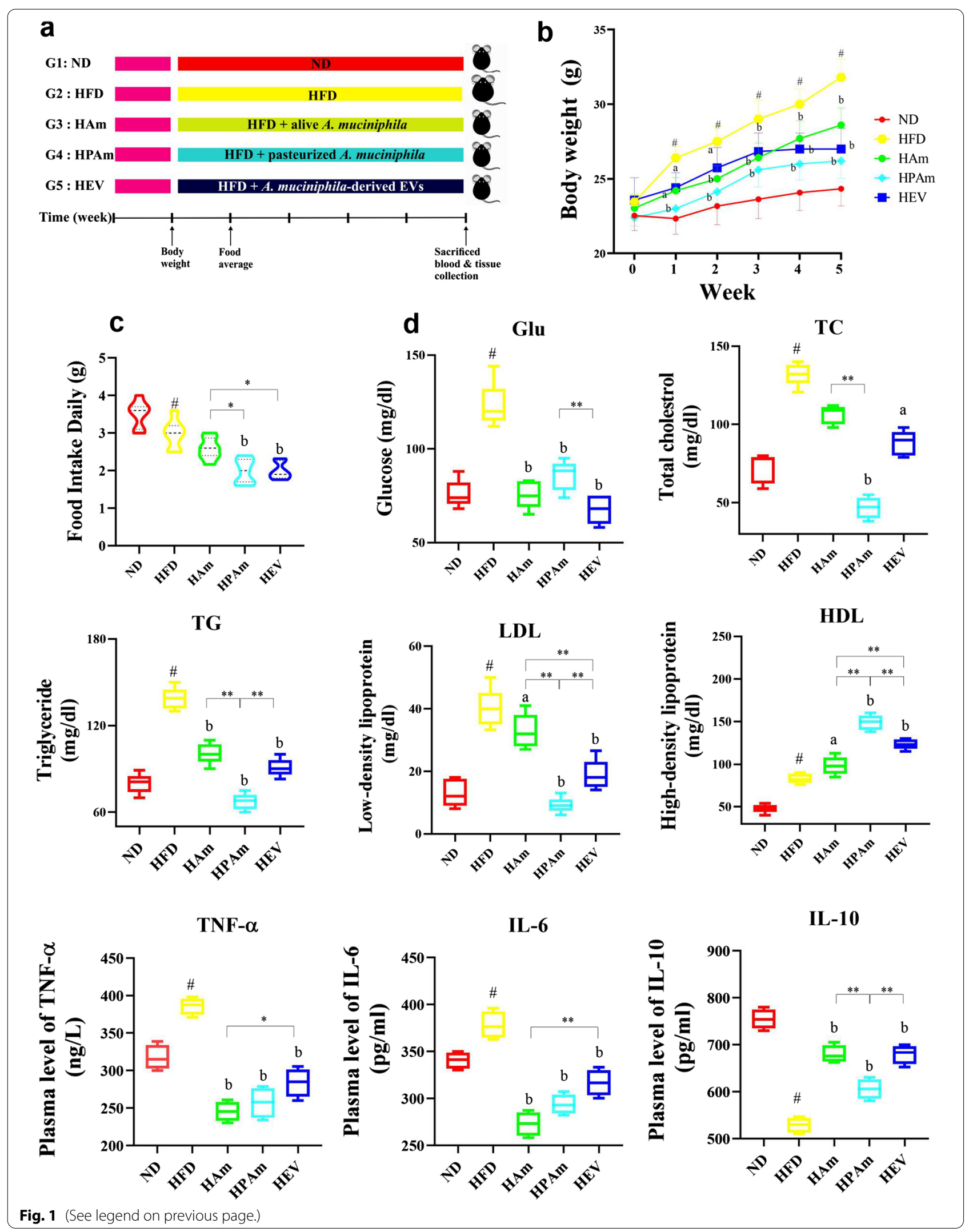




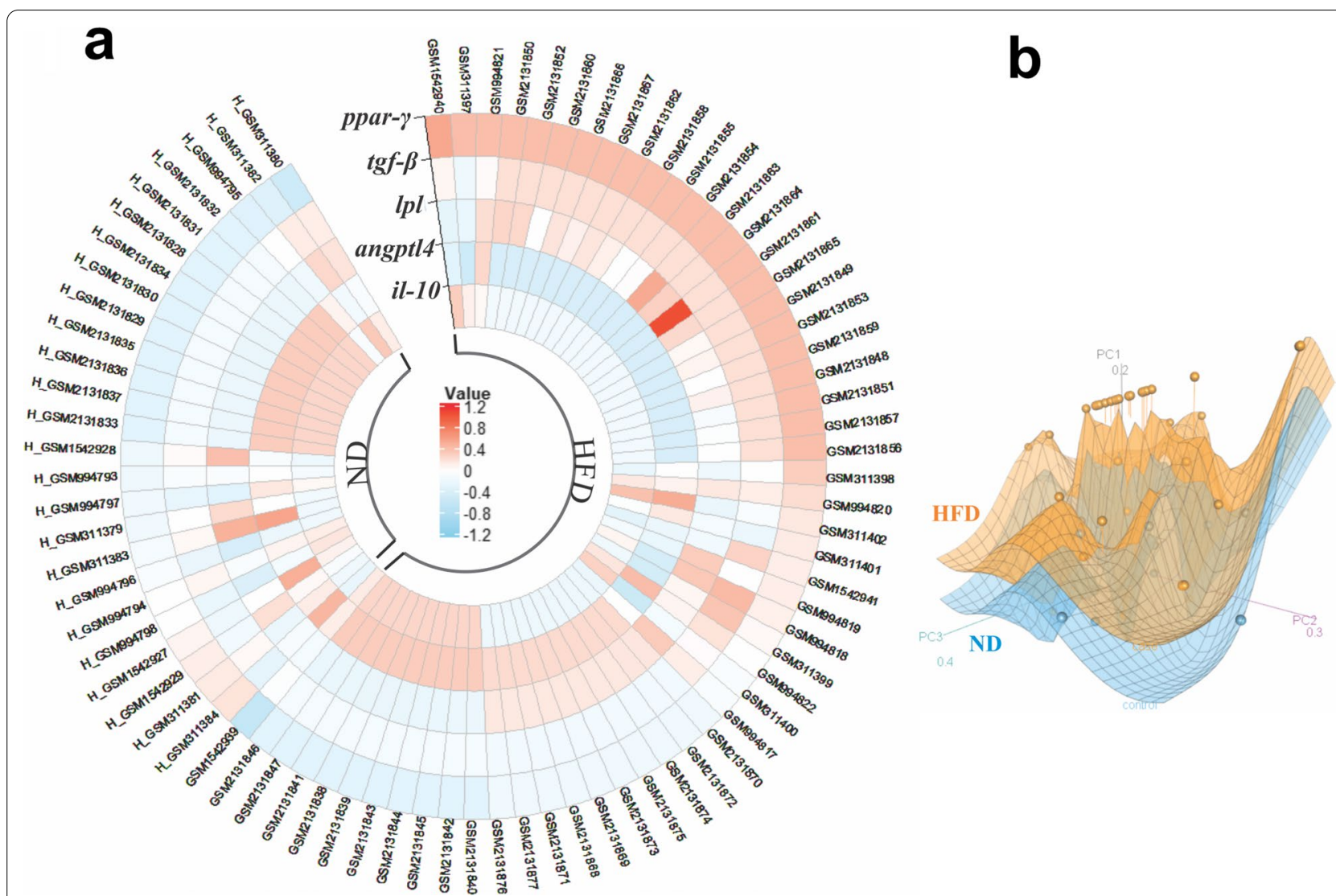

Fig. 2 Heatmap and PCA correlation display different hepatic genes involved in fatty liver between HFD and ND mice. a Heatmap plot of the expression of genes related to HFD-induced fatty liver in HFD- than ND-fed mice. b PCA plot exhibited that the ND group was clustered relatively from the HFD group. HFD and ND group indicated by orange and blue colors respectively

relatively clustered from the HFD group (Fig. 2b). Since these genes, which play a crucial role in lipid metabolism and inflammation, showed similar significant trends in all datasets, they were selected to examine the effects of live and pasteurized A. muciniphila and its EVs on genes associated with HFD-induced fatty liver.

\section{A. muciniphila prevented the onset of fatty liver in HFD-fed mice by modulating lipid metabolism and inflammation}

To examine the effects of live or pasteurized A. muciniphila and its EVs on fatty liver, we assessed the liver histopathology, liver enzymes, and hepatic lipid and inflammation-related genes. In the histological analysis, a higher infiltration of inflammatory cells was observed in the HFD group than the ND group. Although all treatments prevented the HFD-induced inflammation, a few inflammatory cells were found in the HPAm group. Moreover, excessive accumulation of lipid droplets in both macrovesicular and microvesicular forms was found in HFD-exposed hepatocyte cells, while all treatments prevented and reduced the HFD-induced accumulation of lipid droplets; nevertheless, the HPAm group showed few lipid droplets (Fig. 3a) Overall, live A. muciniphila and its EVs exerted better preventive effects on HFDinduced hepatic steatosis and induced a normal morphology similar to the ND group.

HFD enhanced liver weight, however HEV, HPAm, and HAm significantly prevented HFD-induced weight gain. Notably, hepatic weight of HEV and HPAm were lowered, but no significant difference was observed in hepatic weight as compared to HAm group (Fig. 3b). ALT and AST concentrations in the HFD group were highest, while all treatments prevented these alterations, notably, pasteurized A. muciniphila had the most effective reduction in ALT level (Fig. 3c).

Following HFD feeding, the expression of hepatic lipid metabolism-related genes, e.g., ppar- $\gamma$ and $l p l$, were upregulated as well as angptl4 expression was downregulated. Interestingly, all treatments had lipid-associated genes lowering effects. The highest expression level of $\operatorname{tg} f-\beta$ was observed in the HFD group, while all treatments prevented this upregulation; however, the remarkable effect on downregulation of tgf- $\beta$ was observed in the HPAm and HEV as compared to HAm group. In HFD-fed mice, the mRNA 


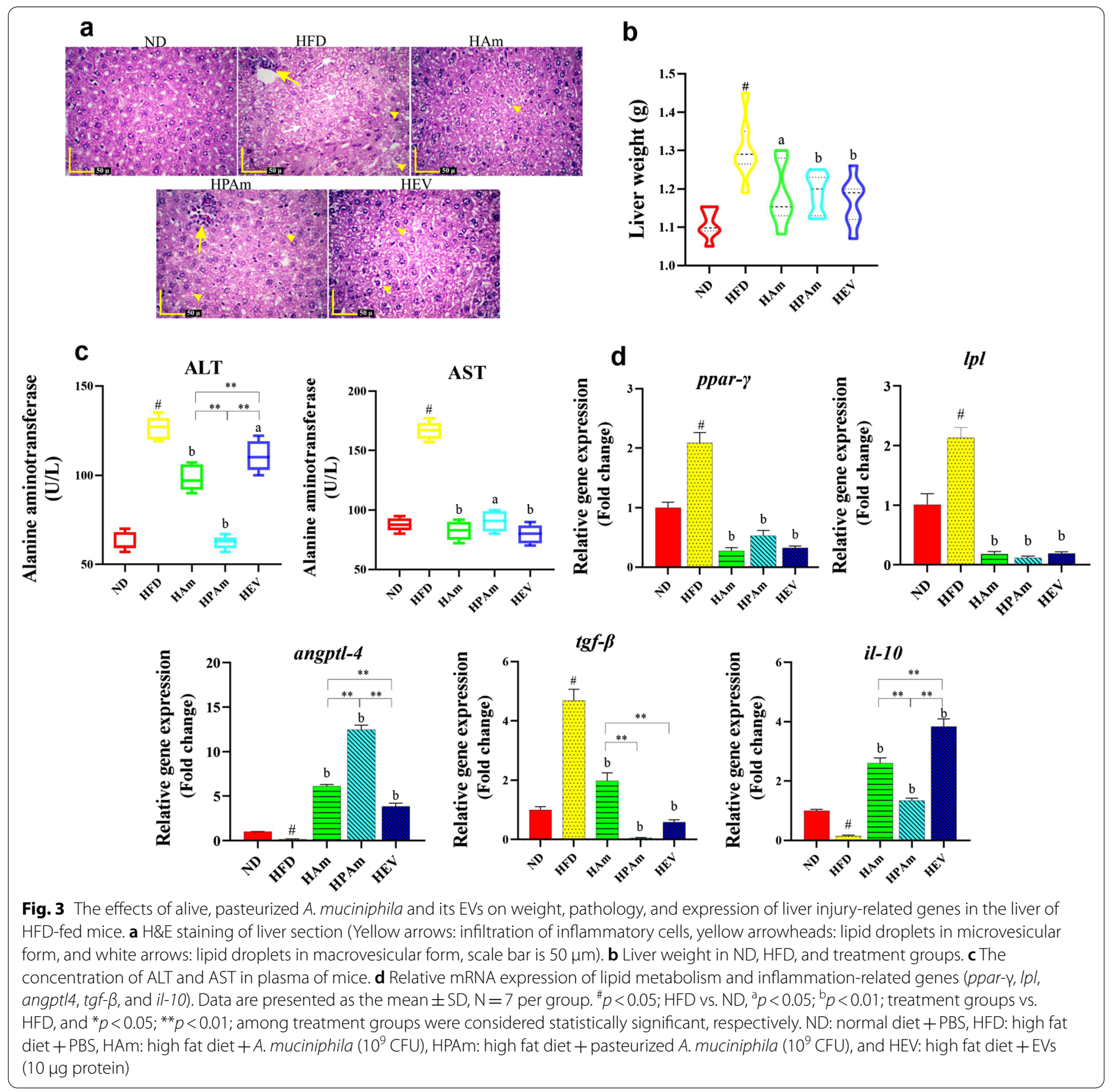

level of $i l-10$ was weakly expressed, however treatments potentially exert anti-inflammatory effect via expression of high il-10 level (Fig. 3d). It is intriguing to note that the EVs showed the highest anti-inflammatory effect. Altogether, these results showed that $A$. muciniphila could prevent HFD-induced fatty liver.

\section{A. muciniphila administration prevented HFD-induced intestinal barrier disturbance and inflammation}

Because HFD induced an alteration in morphology, integrity, and inflammation of HFD-fed mice colon, we evaluated colon tissue by H\&E staining and qPCR. HFD induced focal infiltration of inflammatory cells in the lamina propria and epithelium in the colon; conversely, no inflammatory reactions were present in all treatments. Moreover, the crypt depth and mucous layer thickness showed a considerable decrease in the HFD group, in contrast, all treatments prevented these changes (Fig. 4a).

HFD reduced intestinal integrity by downregulating. $z o 1$, ocldn, and $c l d n-1$ as well as an increase in cldn-2 expression, whereas modulating these genes were observed after all treatments (Fig. 4b). The 


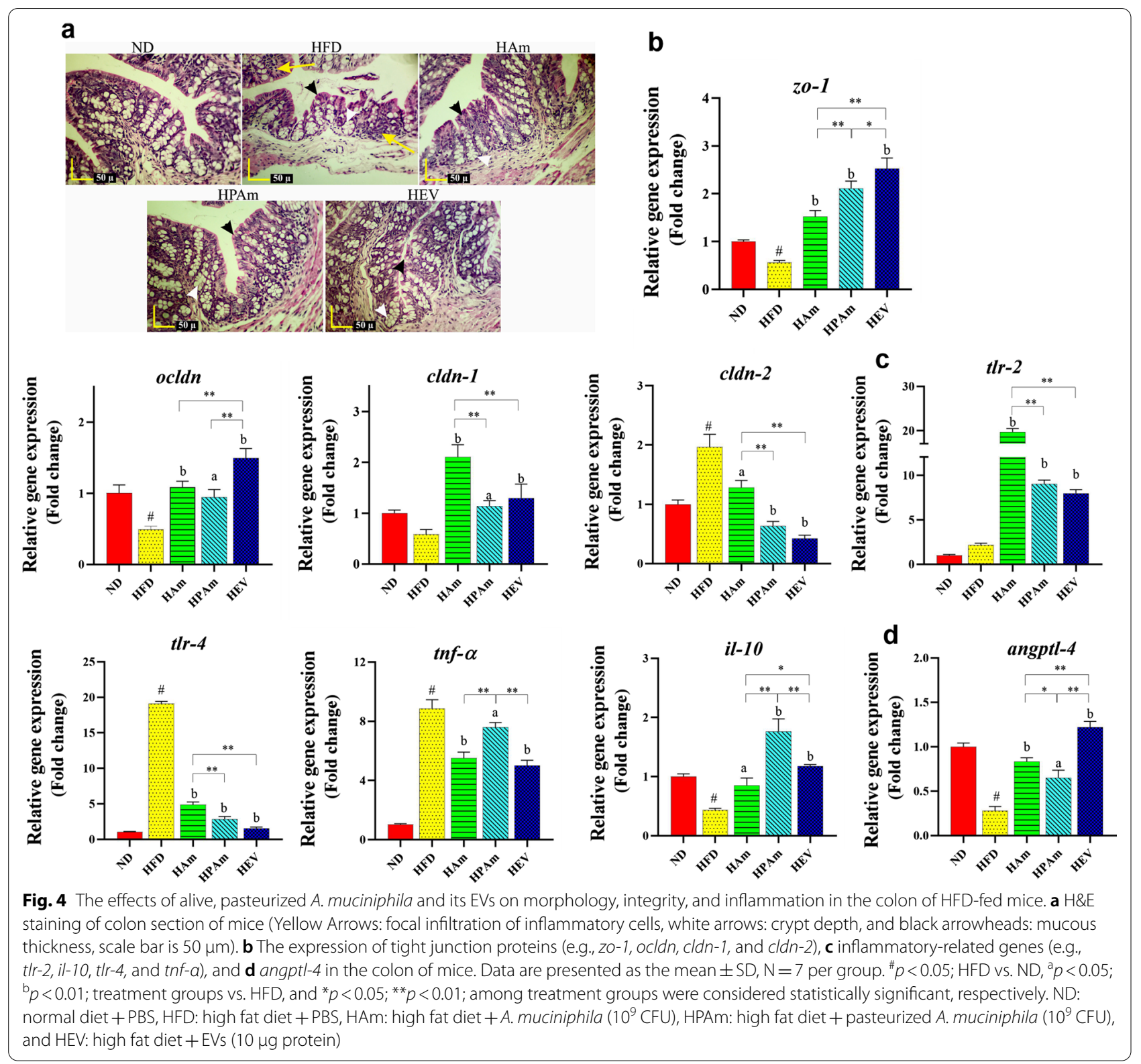

highest increase in $z o-1$ and ocldn was observed in the HEV group, however the greatest upregulation of cldn-1 was seen in the HAm group. Furthermore, more downregulation in $c l d n-2$ was observed in the HEV and HPAm groups. These results showed that the modulation of tight junction genes were responsible for suppressing HFD-induced intestinal permeability induced by all treatments.

HFD upregulated the expression of colonic inflammatory markers, whereas all treatments protected against these changes. These observations were accompanied by upregulating $t l r-2$ and $i l-10$ as well as downregulating $t l r-$ 4 and $\operatorname{tnf}-\alpha$ (Fig. 4c). The EVs had the highest effects on reducing mRNA level of inflammatory genes, while live and pasteurized $A$. muciniphila had more increasing tlr2 and $i l-10$ expression, respectively. These findings indicated that pasteurized A. muciniphila and its EVs had a markedly more influence on the protection against HFDinduced intestinal alterations.

To explore the impact of live and pasteurized A. muciniphila and its EVs on lipid metabolism, we assessed colonic gene expression of Angiopoietin-like 4 (Angptl4). The colonic expression of angptl4 reduced in the HFD group, whereas significantly upregulated in treatment groups, among which the HEV group had a better effect (Fig. 4d). Together, the EVs had notable impacts on 
the prevention of obesity by the modulation of genes involved in adiposity and energy metabolism.

\section{A. muciniphila prevented an increase in adipocyte size, lipid metabolism, and inflammation in adipose tissue of HFD-fed mice}

To evaluate whether the live and pasteurized A. mисіniphila and its EVs had preventive effects on HFD-caused adipose dysfunctions, we assessed eWAT by histopathology analysis, qPCR, and ELISA. The HFD increased the adipocyte size, whereas all treatments exerted preventive effects on this parameter. In the HEV, HPAm, and HAm groups, the adipocyte size decreased; the HEV group exhibited a normal morphology similar to the ND group. Also, HFD increased the infiltration of inflammatory cells in the interstitial tissue than other treatments; however, inflammation was less alleviated in the HAm and HEV groups as compared to the HPAm group (Fig. 5a).

Results of the adipocyte surface area revealed that the HFD group included the maximum average $\left(5613.71 \pm 114.29 \mu^{2}\right)$, compared to that in other groups $(\mathrm{P}<0.001)$. Furthermore, adipocyte surface area showed respectively a significant decrease in HEV $\left(1170.19 \pm 72.38 \mu \mathrm{m}^{2}\right)$, HPAm $\left(2349.31 \pm 33.45 \mu^{2}\right)$ and

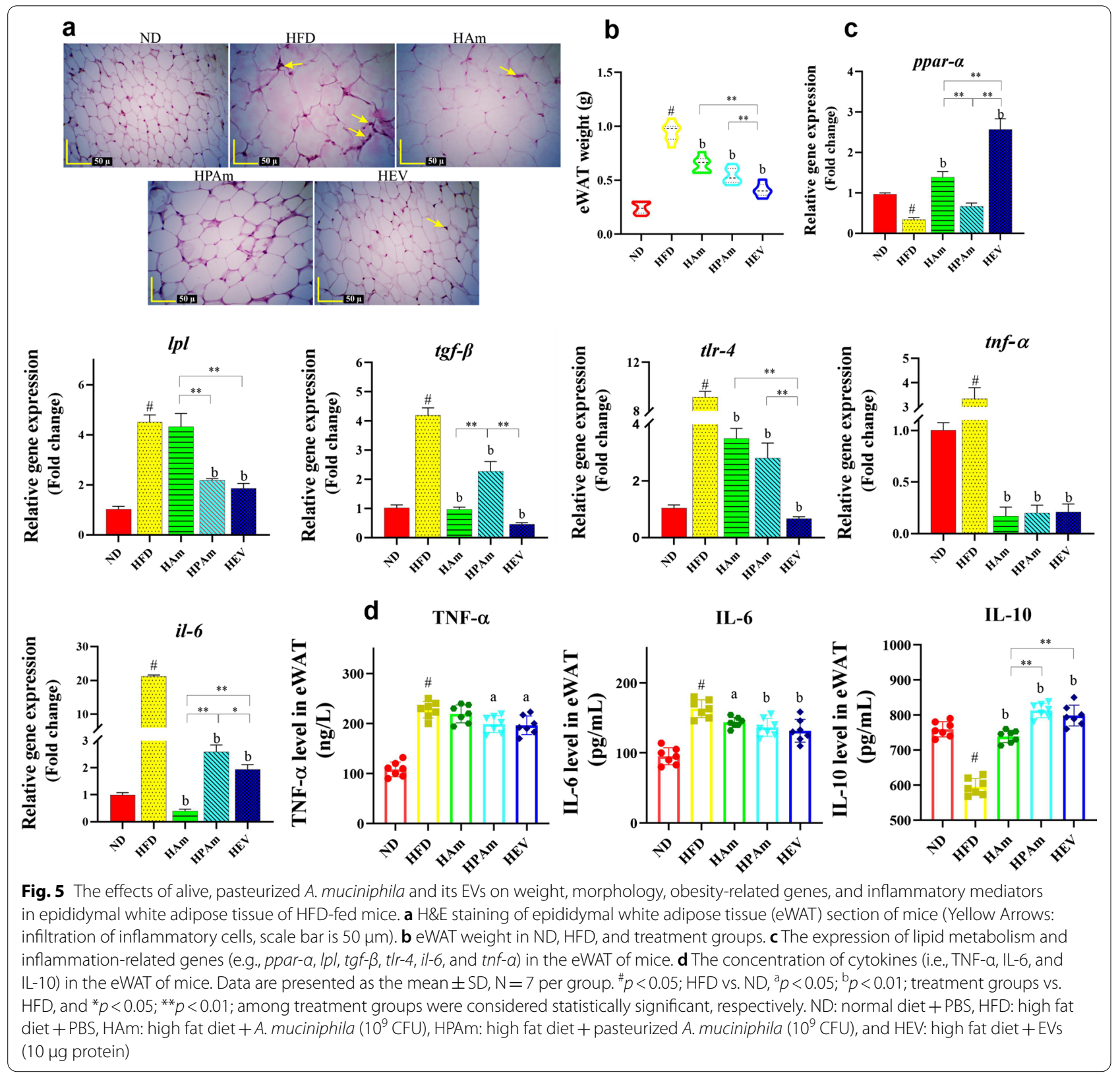


HAm $\left(2452.21 \pm 124.36 \mu \mathrm{m}^{2}\right)$ groups, compared to that in HFD group $(\mathrm{P}<0.001)$ (Additional file 1: Fig. S2).

The HFD group showed a higher eWAT weight than ND-fed mice. However, all treatments significantly prevented this weight gain, the EVs had the greatest effect on it (Fig. 5b). These finding exerted that A. muciniphila and its EVs play a key role in preventing HFD-induced obesity by affecting eWAT weight.

In comparison with normal mice, the expression of lipid oxidation gene i.e. ppar- $\alpha$ was decreased in the eWAT of HFD-fed mice. All treatments could upregulate this expression; however, the HPAm showed no significant change. The EVs had the highest effect on it. Moreover, $l p l$ and $t g f-\beta$ were significantly expressed in the HFD group than the ND group. The HPAm and HEV groups had the same effect on the reduction in $l p l$ expression, but HAm didn't significantly effect on it. The significant downregulation of $\operatorname{tg} f-\beta$ was observed in all treatment groups, while the EVs and live A. muciniphila showed a more noticeable effect.

The overexpression of adipo-inflammatory genes including $t l r-4$, $i l-6$, and $t n f-a$ were observed in HFD-fed mice, while treatments alleviated adipo-inflammation. The same reduction of $t l r-4$ and $t n f-\alpha$ expression were observed in all treatments, also the highest reduction in adipose $i l-6$ mRNA level was observed in the HAm group (Fig. 5c).

Besides, increased TNF- $\alpha$ and IL- 6 level and decreased IL-10 level was also observed in the HFD group, while all treatments prevented HFD-induced adipo-inflammation. Of note, pasteurized A. muciniphila and its EVs induced the highest effects on the concentrations of inflammatory mediators (Fig. 5d). These results suggested that the postbiotic and paraprobiotic A. muciniphila could prevent HFD-induced adipo-inflammation.

\section{A. muciniphila prevented HFD-induced gut dysbiosis by balancing the microbial population}

To explore whether A. muciniphila can modulate HFD-mediated gut microbiota dysbiosis, we quantified the microbial composition in gut of mice through $16 \mathrm{~S}$ rRNA gene-targeted phylum- and group-specific primers (Additional file 1: Table S3) by real-time PCR. HFD significantly augmented Fusobacteria and Firmicutes as well as reduced Bacteroidetes, Actinobacteria, and Verrucomicrobia than the ND group. All treatments affected the gut microbiota composition. The Firmicutes abundance decreased in the HPAm and HEV groups, while the Verrucomicrobia abundance increased in the HAm group (Fig. 6a). On the other hand, pasteurized $A$. muciniphila and its EVs significantly decreased the Firmicutes/Bacteroidetes ratio (Fig. 6b). The heatmap represents a comparison of the relative abundance of bacteria at class, family, and genus levels among the experimental groups (Fig. 6c). There was a significant increase in $\gamma-/ \varepsilon$ - $/ \alpha$-Proteobacteria, Enterobacteriaceae, and Clostridia abundance as well as decreased Ruminococcaceae in the HFD group than the ND group. After five weeks, there was a significant decline in $\alpha$-Proteobacteria in the HPAm group, $\gamma$-Proteobacteria in the HAm and HEV groups, Prevotellaceae in the HPAm and HEV groups, and Clostridia in the HEV group. At genus level, the gut microbiota of the HFD group contained the highest level of Roseburia spp., Enterococcus spp., Lactobacillus spp., and E. coli and the lowest level of Veillonella spp., Alistipes spp., Bifidobacterium spp., Methanobrevibacter spp., and A. muciniphila. Interestingly, live $A$. muciniphila was the most effective in preventing these HFD-induced dysbiosis, which was accompanied by increasing Alistipes spp. and $A$. muciniphila and decreasing Roseburia spp. and E. coli. Pasteurized A. muciniphila also significantly reduced the abundance of Roseburia spp. Overall, A. muciniphila could avoid HFD-induced dysbiosis by decreasing obesity-related pathobiont bacteria and increasing health-related gut microbiota.

\section{Effects of $A$. muciniphila on correlation between the gut microbiota abundance and obesity-related indices}

The correlations between gut microbiota and obesityrelated indices demonstrated that Firmicutes was negatively correlated with adipose IL-10 level and colonic il-10 expression, whereas it had positive association with hepatic tgf- $\beta$ expression. Bacteroidetes level was negatively correlated with the eWAT weight, the adipose level of TNF- $\alpha$ and IL6, the expression of adipose $l p l$, and colonic $t l r-4$. There was reverse correlation between body weight and Fusobacteria abundance, which had the highest relative abundance in the HFD group. Verrucomicrobia abundance was a negative correlation with adipose il-6 mRNA level. Besides the liver weight, the colonic tnf- $\alpha$ mRNA level had a significant positive association with the abundance of Enterobacteriaceae and Lactobacillus spp., while the abundance of these bacteria had a negative association with the plasma IL-10 level. Moreover, the Clostridia abundance was positively associated with the adipose $t l r-4$ mRNA level, while $A$. muciniphila had a negative correlation with the il-6 expression in the eWAT. ALT was positively correlated with pathobiont bacteria, such as Roseburia spp. and $\alpha$-Proteobacteria (Fig. 6d). These findings revealed that the administration of $A$. muciniphila could play a beneficial role in the modulation of host responses by affecting the HFD-induced intestinal dysbiosis. 


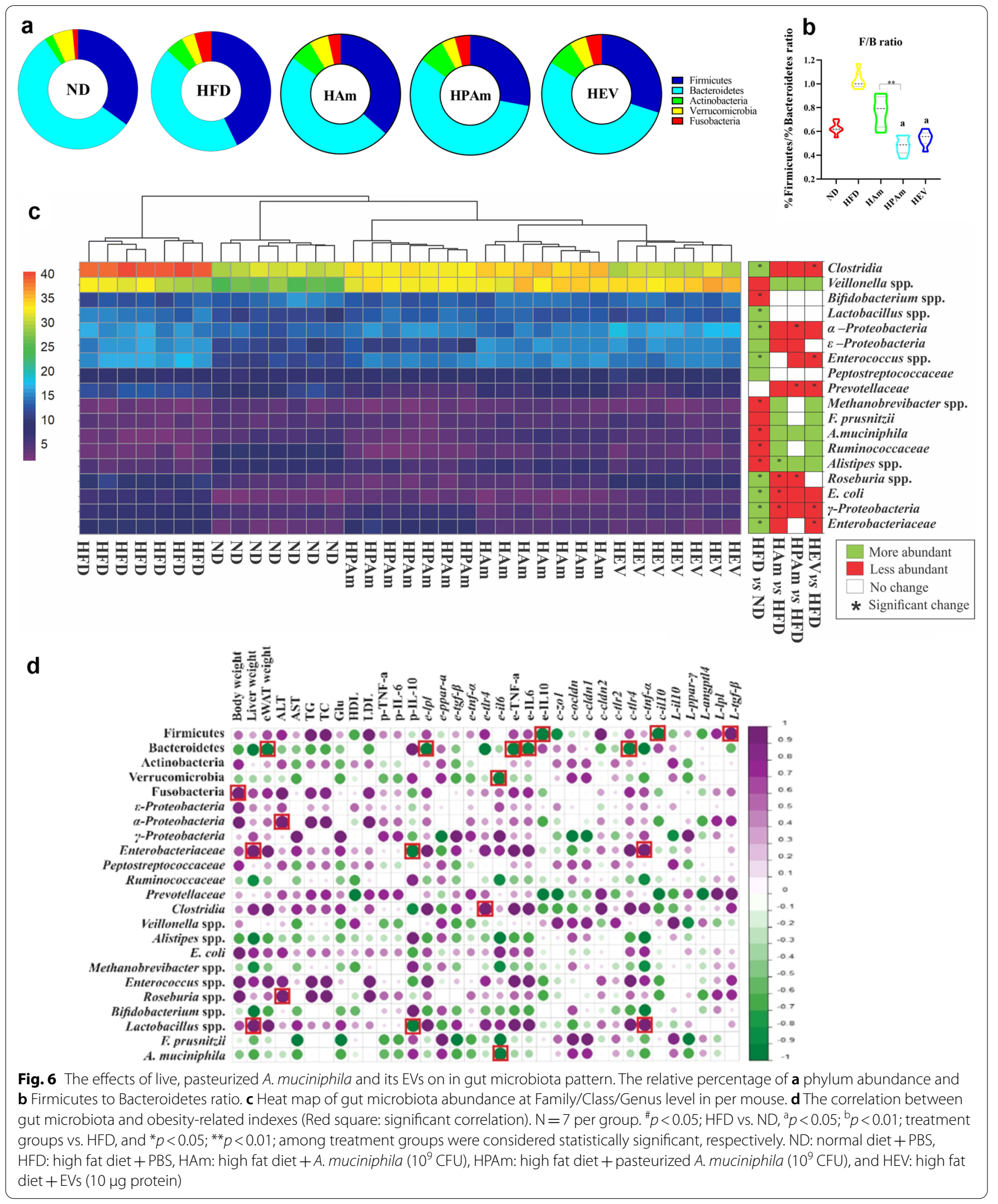




\section{Discussion}

Numerous studies have indicated that several probiotics can prevent HFD-induced obesity [5, 11, 12, 15], while the exact mechanism of probiotics remains unclear. In this study, we carried out a comparative analysis of the effects of live and pasteurized A. muciniphila and its EVs on the HFD-fed mice to understand the possible mechanisms underlying their preventive effects on obesity.

Functional studies have reported that HFD increases the body weight, metabolic tissue weight, adipocyte size, and hepatic lipid droplets. On the other hand, probiotics can inhibit the weight gain and other HFD-induced effects $[16,25]$. We found that all treatments reduced the body, eWAT, and liver weight gain, in addition to the adipocyte size and hepatic lipid droplets in HFD-fed mice; however, the efficacy of treatments varied. Pasteurized A. muciniphila and its EVs showed greater effectiveness than other treatments. Similarly, the effects of A. muciniphila and its EVs in HFD-fed mice revealed that this bacterium and its EVs could ameliorate obesity by reducing the body weight gain, fat accumulation, and pathological abnormalities [14, 15, 22]. Besides, an earlier study demonstrated that pasteurized $A$. muciniphila had greater effects on the fat mass loss and obesity amelioration compared to its live form [16]. Since the gut microbiota is critical for the management of body weight, our results showed that probiotics, paraprobiotics, and postbiotics may have potential effects on preventing diet-induced obesity.

Besides, probiotics can affect the plasma lipid profile and inflammatory cytokines and ameliorate obesity in HFD-fed animals [15, 26]. We found that all treatments improved the metabolic parameters and prevented the onset of obesity; notably, pasteurized A. muciniphila showed greater effects on these parameters. In agreement with our study, previous studies revealed that administration of live and pasteurized $A$. muciniphila could reduce the level of cholesterol in animal and human models $[16,27]$. Moreover, we reported the reducing effects of A. muciniphila and its EVs on the metabolic parameters in obese mice [15]. These findings indicated that both forms of A. muciniphila and its EVs play a preventive role in the onset of HFD-induced obesity and its related complications.

Substantial evidence corroborates that obesity is associated with intestinal dysfunction and low-grade inflammation. Therefore, regulation of obesity-related inflammation and restoration of the impaired intestinal barrier function have been proposed as potential therapeutic targets in the past few decades $[28,29]$. Our findings indicated the greatest increase in the intestinal permeability and inflammatory mediators, besides the lowest mucus thickness and crypt depth in the HFD group, while all treatments prevented these changes. The mechanism of HFD includes reducing intestinal integrity, penetrating LPS into lamina propria, stimulating the immune system, and inducing low-grade inflammation [30]. Thus, all treatments prevented HFD-induced morphological changes by decreasing gut permeability and ameliorating LPS-induced inflammation.

Remarkably, the pasteurized A. muciniphila and its EVs completely suppressed the HFD-induced intestinal inflammation in HFD-fed mice and protected the intestinal permeability. In this regard, previous studies on pasteurized and live A. muciniphila showed that HFDinduced intestinal permeability was alleviated following both treatments by affecting the expression of tight junction components [16] and colonic morphological features [14, 31]. Moreover, research on A. muciniphila EVs demonstrated their beneficial effects on the obesity-induced gut permeability [15, 22], DSS-induced colitis [21], and Caco-2 cell line [32]. Overall, these findings suggest that probiotics can strengthen the intestinal barrier integrity, reduce inflammation, and consequently, prevent metabolic disorders, which might be due to a close link between probiotics and intestinal cells.

In addition, we observed that all treatments significantly upregulated the colonic expression of Angptl-4, while HFD suppressed it. Similarly, it has been shown that A. muciniphila and its EVs, in addition to Lactobacillus rhamnosus CNCMI-4317, upregulated Angptl-4 in obese mice and the colon cell line, respectively [15, 33]. Therefore, probiotics and their vesicles may have positive effects on the intestinal homeostasis and may prevent obesity.

According to previous studies, the HFD-induce leaky gut is strongly linked to key metabolic dysfunctions in the white adipose tissue by triggering inflammation and lipid metabolism; also, lipid is redistributed in ectopic organs [14, 34]. Our results indicated that HFD increased the eWAT weight, inflammation, and energy homeostasis disturbances; conversely, all treatments protected these alterations. The EVs showed the greatest effects on the adipocyte size, eWAT weight, and lipid homeostasis, compared to the other treatments. Besides EVs, pasteurized A. muciniphila had remarkable effects on the adipose inflammatory mediators. It has been shown that A. muciniphila improves the metabolic status in obese mice by reducing the expression [31] and production [35] of adipose inflammatory cytokines. During obesity, adipose tissue remodeling occurs, which causes adipocyte enlargement, extracellular matrix components accumulation, and infiltration of pro-inflammatory macrophages, thereby modulating adipose tissue remodeling could prevent obesity [36]. Our finding demonstrated that all treatments reduced adipo-inflammation and adipocyte size, 
which may be related to the prevention of HFD-induced adipose tissue remodeling in obese mice.

Recent investigations have confirmed the regulatory effects of A. muciniphila on energy homeostasis in obese mice by reducing the energy absorption and increasing the fecal energy excretion [16, 17]. Besides, pasteurized A. muciniphila could modulate the fat mass and prevent diet-induced obesity [16]. These findings indicate that active and inactive probiotics and their derivatives can prevent HFD-induced inflammation and maintain the energy balance. This finding supports the potential health benefits of probiotic, paraprobiotic, and postbiotic $A$. muciniphila in the prevention of obesity.

HFD, by increasing the intestinal permeability, leads to the transfer of bacterial LPS and other components to the liver through the portal vein and results in hepatic inflammation [9]. Also, the HFD-induced adipocyte dysfunction increases the free fatty acids export from the adipose tissue to the liver and leads to fatty liver development [37]; however, probiotics can prevent and ameliorate non-alcoholic fatty liver disease (NAFLD) [38]. In this study, we found a significant increase in the plasma concentrations of ALT and AST, liver weight, fat content, and inflammatory status in the liver sections of HFDinduced mice; nevertheless, all treatments alleviated these liver alterations. It seems that live and pasteurized A. muciniphila reduce the level of liver injury-related enzymes in obese mice and individuals [27, 39, 40]; the EVs and pasteurized form of $A$. muciniphila also showed more significant effects on the prevention of fatty liver. These findings support the hypothesis that non-viable probiotics can be more effective in counteracting obesityrelated fatty liver disease due to a HFD-related leaky gut.

Generally, HFD causes changes in the expression of hepatic genes involved in lipid metabolism and inflammation. Moreover, the association of $l p l$ overexpression and accumulation of liver triglycerides with NAFLD has been shown in the literature, while the overexpression of hepatic Angptl4 seems to inhibit LPL in peripheral tissues $[41,42]$ and play an important role in the protection of fat accumulation [43] and FA-related inflammation [44]. Our results, in line with our meta-analysis, showed that HFD changed the expression of fatty liverrelated genes by upregulating ppar- $\gamma, l p l$, and $t g f-\beta$ and downregulating angptl4 and $I L-10$ in the liver of mice; on the other hand, all treatments could modulate their expression.

According to a previous study, probiotic supplementation reduced the hepatic and adipose $l p l$ expression in HFD-fed mice and ameliorated obesity [45]. In the present study, we found the beneficial effects of all treatments on preventing fatty liver, as indicated by the improved lipid metabolism, inflammation, and fibrosis genes in the liver. Consistent with our findings, $A$. muciniphila had protective effects against fatty liver by reducing the FA synthesis and inflammation in the liver of obese mice [46]. Moreover, animal and cell line studies demonstrated that live and heat-killed probiotics can reduce the level of hepatic inflammatory and fibrotic genes $[47,48]$. These findings revealed that probiotic, postbiotic, and paraprobiotic $A$. muciniphila had protective effects against fatty liver disease, probably through the MAMPs and bioactive components.

Several studies have shown a strong interaction between the gut microbiota disruptions and the onset of obesity $[49,50]$. The increased abundance of Firmicutes and the decreased level of Bacteroidetes have been reported in obese mice [51], which suggests a possible link between Firmicutes and increased calorie intake [52]. We found the increased level of Firmicutes and the reduced level of Bacteroidetes in the HFD group, while the Firmicutes abundance decreased in both HPAm and HEV groups. In line with other studies [53, 54], we found that the Firmicutes/Bacteroidetes (F/B) ratio remained unchanged in the HFD and ND groups, while a higher $\mathrm{F} / \mathrm{B}$ ratio in obese compared to lean individuals were reported in other studies [55, 56]. According to controversial data about this ratio, it is difficult to link the ratio with determining health status and consider it as a hallmark of obesity [57]. In our study, all treatments decreased this ratio, although it was more significant in the HPAm and HEV groups. Moreover, recent investigations have demonstrated that Actinobacteria and Verrucomicrobia have negative and positive correlations with obesity, respectively [58, 59]. We also showed similar results in the HFD group, while a significant increase in the Verrucomicrobia abundance was seen in the HAm group.

In the present study, the abundance of some pathobiont bacteria, such as the $\gamma / \varepsilon / \alpha$-proteobacteria, Enterobacteriaceae, Prevotellaceae, and Clostridia, increased in the HFD group, while the treatments could reduce their abundance. The high abundance of harmful bacteria has been also reported in obese patients [60]. On the contrary, the abundance of Prevotellaceae seems to reduce in compound probiotic-treated rats [61], which is similar to our results in the HPAm and HEV groups. Moreover, the decreased level of Enterobacteriaceae was reported in Lactobacillus paracasei HIIO-treated obese rats [62]. These observations demonstrated that HFD might cause an imbalance in the gut microbiota, increase the level of harmful bacteria, and consequently increase LPS-induced inflammation; on the other hand, probiotics could modulate these alterations.

Many studies have reported a reduction in the abundance of Ruminococcaceae, Bifidobacterium spp., $F$. 
prausnitzii, and A. muciniphila as beneficial bacteria in the HFD group [63,64]. Similarly, we found the same reduction in the HFD group, although the level of $A$. muciniphila increased in the HAm group. These findings revealed the beneficial effects of probiotics on the restoration of health-promoting microbiota. In this regard, a human study indicated a significant decline in the abundance of Alistipes spp. and an increase in Roseburia spp. in obese individuals [65]. We found similar results in the HFD group, whereas both forms of A. muciniphila normalized the level of Roseburia spp. close to the ND group. In this study, the Firmicutes abundance was negatively correlated with the adipose $i l-10$ level and colonic il-10 expression, while it was positively associated with the hepatic expression of tgf- $\beta$. Similar to our results, a positive correlation between Firmicutes and inflammation was observed in a previous study [66], which was due to SCFA hydrolysis by Firmicutes-secreted enzymes [67]. The increased abundance of Fusobacteria and decreased Bacteroidetes were significantly associated with the body weight and eWAT weight, respectively.

Moreover, the level of Bacteroidetes was negatively correlated with colonic inflammation, pro-inflammatory cytokines, and lipid metabolism in the eWAT. Similarly, an inverse correlation have been reported between the level of Bacteroidetes and body fat gain [68] and inflammatory mediators [66]. The negative correlation with inflammation could be due to secreting SCFA (acetate and propionate) [69] or its polysaccharides [70]. Also, the abundance of Enterobacteriaceae and Lactobacillus spp. was positively correlated with the liver weight and colonic inflammation; conversely, it had a negative correlation with the plasma anti-inflammatory cytokines. The adipose inflammation was positively correlated with the abundance of Clostridia and negatively associated with the abundance of Bacteroidetes, Verrucomicrobia, and $A$. muciniphila.

Based on the present findings, the concentration of ALT was positively associated with the abundance of pathobiont bacteria, such as $\alpha$-proteobacteria and Roseburia species. In this regard, several studies have shown a correlation between the increased level of pathobiont bacteria in the gut and obesity-related indices [49, 71, 72]. These observations reveal that changes in the gut microbiota pattern have destructive effects on metabolicrelated biomarkers and body weight, while preventing these alterations by probiotics, postbiotics, and paraprobiotics may be promising for the prevention of obesity.

\section{Conclusion}

The administration of live and pasteurized A. muсiniphila and its EVs could prevent several complementary mechanisms involved in obesity, such as increased obesity-related indices, elevated inflammatory status in the HFD-fed mice. Notably, pasteurized A. muciniphila and its EVs are more likely to be accounted for the preventive effects than its live form. All treatments could also modulate the relative abundance of some genera (by increasing beneficial microbiota and inhibiting the growth of pathobiont bacteria) and maintain a healthy intestinal homeostasis, reduce obesity, and promote health. The present findings suggest that pasteurized $A$. muciniphila as a paraprobiotic agent and its EVs as postbiotic agents can be new preventive strategies against obesity.

\section{Methods}

\section{Bacterial strains and culture conditions}

A. muciniphila MucT (ATCC BAA-835) was grown in a basal mucin-based medium as previously described [73]. After the bacterial density reached an $\mathrm{OD}_{600}$ of 1 , the pellets were removed by centrifugation, washed twice, and re-suspended with anaerobic PBS. The remaining supernatant was used for EVs extraction. Pasteurization of $A$. muciniphila was performed at $70{ }^{\circ} \mathrm{C}$ for $30 \mathrm{~min}$.

\section{Preparation of A. muciniphila EVs}

Following filtering of the supernatant, EVs were extracted in an ultracentrifuge (Beckman, Germany) at $200000 \mathrm{~g}$ for $2 \mathrm{~h}$ at $4{ }^{\circ} \mathrm{C}$ [21]. The final pellets were re-suspended in PBS and stored at $-80{ }^{\circ} \mathrm{C}$. The morphology assessment of EVs and molecular weights of proteins were performed using Scanning Electron Microscopy (SEM) and SDSPAGE, respectively, as detailed in the Additional file 1: Additional methods.

\section{Animal and experimental setup}

All animal experiments were performed in accordance with the Animal Experiment Committee of Pasteur Institute of Iran guidelines for the care and use of laboratory mice. For the prevention study, 35 eight-week-old male C57BL/6 mice were obtained from the Pasteur Institute of Karaj (Iran). Mice were individually housed with ad libitum access to food and autoclaved water.

$\left(22-24{ }^{\circ} \mathrm{C}, 40-60 \%\right.$ humidity, and $12 \mathrm{~h}$ light/dark). After one week of acclimation with normal diet (ND) (A03, safe diet, France), mice were randomly separated into five groups $(n=7)$, based on best results of our previous study [15]. As shown in Fig. 1a, mice were fed HFD (260 HF, 60\% energy from butter (Kcal/kg), safe diet, France) along with treatments for five weeks includes: (1) ND (standard diet $+200 \mu \mathrm{l}$ PBS) (as control); (2) HFD (HFD + $200 \mu \mathrm{l} \mathrm{PBS)} \mathrm{(as} \mathrm{control);} \mathrm{(3)} \mathrm{HAm}$ (HFD $+10^{9} \mathrm{CFU} / 200 \mu$ live A. muciniphila); (4) HPAm (HFD $+10^{9} \mathrm{CFU} / 200 \mu \mathrm{l}$ pasteurized $A$. muciniphila); and (5) $\mathrm{HEV}(\mathrm{HFD}+10 \mu \mathrm{g}$ protein/200 $\mu \mathrm{l} \mathrm{EVs)}$. Body weight 
was measured weekly and food was monitored daily. Blood, epididymal adipose (eWAT), liver, and colon tissues were collected and stored at $-80{ }^{\circ} \mathrm{C}$. In addition, eWAT, liver, and colon are saved for histological analysis.

\section{Serological and histological analysis}

Fasting blood glucose (Glc), total cholesterol (TC), low-density lipoprotein (LDL), high-density lipoprotein (HDL), triglyceride (TG), alanine aminotransferase (ALT), and aspartate aminotransferase (AST) levels were measured using a commercial kit (Bioclin-Quibasa, Brazil). Moreover, TNF- $\alpha$, IL-6, and IL-10 cytokines level in plasma and eWAT were quantified by using ZellBio GmbH ELISA kit (Germany) according to the manufacturer's instructions. For histological evaluation, the tissues were stained with hematoxylin and eosin (H\&E). Dino-lite digital lens, Dino Capture 2 software (AnMo Electronics Corp., Taiwan), and light microscope were used for histopathological analysis, as detailed in the Additional file 1: Additional methods. All test was performed in duplicate.

\section{Computation of selected gene expression}

The National Centre for Biotechnology Information Gene Expression Omnibus (GEO) was used to download gene expression datasets. Our inclusion criteria for accessing appropriate datasets as follow: Studies in which: (1) mice fed HFD for 4-6 weeks; (2) the number of samples for each group (i.e., ND and HFD) must be more than one; (3) using liver tissue; and (4) assessed the expression of all selected genes. A total of four datasets (Additional file 1: Table S1) were obtained that qualified for our study: The $R$ (version 3.6.1) statistical computing environment and BioConductor (version 3.12) were used for gene microarray analyses. Prior to meta-analysis, each dataset was normalized and subsequently transformed with base 2 $\log$. Based on the annotation table for each respective dataset, the probeset identifiers converted into their corresponding Gene Symbol. For multiple probes matching to the same gene, the mean factor of the probes was considered as the final expression value of the gene. Data merging has conducted to correct batch effects using ComBat function of SVA package [74]. Then, we survey the expression of all selected genes across the four datasets by using limma package (version 3.40.6).

\section{Analysis of target genes in colon, adipose, and liver by quantitative real-time PCR}

Total RNA was isolated by Trizol reagent (Bio Basic, Canada). The gDNA was removed by DNase I (Qiagen) then RNA was reverse transcribed using PrimeScript RT Reagent Kit (Takara). Real-time PCR was performed using SYBR Premix Ex Taq II (Takara). The target gene expression was normalized to the housekeeping gene rpl-19 in colon and liver and hprt-1 in adipose tissue. A sequence of primers used in this study is shown in Additional file 1: Table S2.

\section{Analysis of gut microbiota abundance in stool by quantitative real-time PCR}

Fecal DNA were extracted using a QIAamp Fast DNA Stool Mini Kit (Qiagen, USA) according to the manufacturer's instructions. Real-time PCR was perfomed by RealQ Plus Master Mix Green (Amplicon, Denmark). The $\Delta C_{T}$ method was used to measure each primer efficiency [30]. Conversion of $C_{T}$ value to bacterial communication percentage was performed by using percentage formula as previously described [75]. A sequence of primers used in this experiment is shown in Additional file 1: Table S3.

\section{Statistical analysis}

Statistical analysis was performed by GraphPad Prism 8.0 (GraphPad Software Inc, CA, USA), the one-way ANOVA followed by Tukey's post hoc test and KruskalWallis test was used where needed. Moreover, the twoway ANOVA test with a Tukey post-hoc test was used to evaluate the effect of treatments on body weight. The correlations between the gut microbiota and obesityrelated indices were evaluated by Spearman's correlation test. Results are presented as the mean \pm standard error and P-value of less than 0.05 was regarded as statistically significant.

\begin{abstract}
Abbreviations
HFD: High fat diet; ND: Normal diet; EVs: Extracellular vesicles; ANOVA: Analysis of variance; AST: Aspartate aminotransferase; ALT: Alanine aminotransferase; TG: Triglyceride; TC: Total cholesterol; LDL: Low-density lipoprotein; HDL: High-density lipoprotein; FA: Fatty acid; Glc: Glucose; eWAT: Epididymal white adipose tissue; GEO: Gene expression omnibus; TNF: Tumor necrosis factor; IL: Interleukin; TGF: Transforming growth factor; ZO1: Zonula occludens 1; OCLDN: Occludin; CLDN: Claudin; TLR: Toll-like receptor; PPAR: Peroxisome proliferator-activated receptors; Angptl4: Angiopoietin-like 4; LPL: Lipoprotein lipase; H\&E: Hematoxylin and eosin; NAFLD: Non-alcoholic fatty liver disease.
\end{abstract}

\section{Supplementary Information}

The online version contains supplementary material available at https://doi. org/10.1186/s12934-021-01709-w.

Additional file 1. Tables S1, S2, and S3. Fig. S1 and S2. Additional methods.

\section{Acknowledgements}

We would like to acknowledge the cooperation of staff member of Mycobacteriology and Pulmonary Research Department of Pasteur Institute of Iran for their kind cooperation. We thank prof. Willem de Vos for valuable comments that improved this manuscript. 


\begin{abstract}
Authors' contributions
FA designed the study, performed research, interpreted data, and wrote the manuscript. SK performed experimental tests, revised the manuscript, and draw image. AL performed bioinformatics and meta-analyses. AS, SA, MM, and $M D$ performed experimental test. HRM performed dissection of the mice and histopathological evaluation; SDS, FV and SKH designed the study and reviewed/edited the manuscript. MDO and AM reviewed/edited the manuscript. All authors read and approved the final manuscript.
\end{abstract}

\section{Funding}

This research did not receive any specific grant from funding agencies in the public, commercial, or not-for-profit sectors.

\section{Availability of data and materials}

All data generated or analyzed during this study are included in this published article and its additional file..

\section{Declarations}

\section{Ethics approval and consent to participate}

All animal experiments were performed in accordance with the Animal Experiment Committee of Pasteur Institute of Iran guidelines for the care and use of laboratory mice (IR.PII.REC.1395.010).

\section{Consent for publication}

Not applicable.

\section{Competing interests}

The authors declare that they have no conflict of interest.

\section{Author details}

'Microbiology Research Center (MRC), Pasteur Institute of Iran, Tehran, Iran. ${ }^{2}$ Clinical Research Department, Pasteur Institute of Iran, Tehran, Iran. ${ }^{3}$ Systems Biomedicine Unit, Pasteur Institute of Iran, Tehran, Iran. ${ }^{4}$ Basic and Molecular Epidemiology of Gastrointestinal Disorders Research Center, Research Institute for Gastroenterology and Liver Diseases, Shahid Beheshti University of Medical Sciences, Tehran, Iran. ${ }^{5}$ Department of Basic Sciences, School of Veterinary Medicine, Shiraz University, Shiraz, Iran. ${ }^{6}$ Department of Mycobacteriology and Pulmonary Research, Pasteur Institute of Iran, Tehran, Iran. ${ }^{7}$ Department of Biochemistry, Pasteur Institute of Iran, Tehran, Iran. ${ }^{8}$ Department of Medical Genetics, Faculty of Medicine, Shahid Beheshti University of Medical Sciences, Tehran, Iran. ${ }^{9}$ Research Laboratories, Children's Hospital Bambino Gesù-IRCCS, Rome, Italy.

Received: 29 July 2021 Accepted: 16 November 2021

Published online: 04 December 2021

\section{References}

1. Ng M, Fleming T, Robinson M, Thomson B, Graetz N, Margono C, et al. Global, regional, and national prevalence of overweight and obesity in children and adults during 1980-2013: a systematic analysis for the Global Burden of Disease Study 2013. The lancet. 2014;384(9945):766-81.

2. Saklayen MG. The global epidemic of the metabolic syndrome. Curr Hypertens Rep. 2018;20(2):12.

3. Halford JC. Pharmacotherapy for obesity. Appetite. 2006;46(1):6-10.

4. Krentz A, Fujioka K, Hompesch M. Evolution of pharmacological obesity treatments: focus on adverse side-effect profiles. Diabetes Obes Metab. 2016;18(6):558-70.

5. Vallianou N, Stratigou T, Christodoulatos GS, Tsigalou C, Dalamaga M. Probiotics, prebiotics, synbiotics, postbiotics, and obesity: current evidence, controversies, and perspectives. Curr Obes Rep. 2020. https://doi.org/10 1007/s13679-020-00379-w.

6. Plaza-Diaz J, Ruiz-Ojeda FJ, Gil-Campos M, Gil A. Mechanisms of action of probiotics. Adv Nutr. 2019;10(suppl_1):S49-66.

7. Mazloom K, Siddiqi I, Covasa M. Probiotics: how effective are they in the fight against obesity? Nutrients. 2019;11(2):258.
8. Torres S, Fabersani E, Marquez A, Gauffin-Cano P. Adipose tissue inflammation and metabolic syndrome. The proactive role of probiotics. Eur J Nutr. 2019;58(1):27-43

9. Lian C-Y, Zhai Z-Z, Li Z-F, Wang L. High fat diet-triggered non-alcoholic fatty liver disease: a review of proposed mechanisms. Chem Biol Interact. 2020. https://doi.org/10.1016/j.cbi.2020.109199.

10. Herrema H, IJzerman RG, Nieuwdorp M. Emerging role of intestinal microbiota and microbial metabolites in metabolic control. Diabetologia. 2017:60(4):613-7.

11. Wang P, Gao X, Li Y, Wang S, Yu J, Wei Y. Bacillus natto regulates gut microbiota and adipose tissue accumulation in a high-fat diet mouse model of obesity. J Funct Foods. 2020;68: 103923.

12. Munukka E, Rintala A, Toivonen R, Nylund M, Yang B, Takanen A, et al. Faecalibacterium prausnitzii treatment improves hepatic health and reduces adipose tissue inflammation in high-fat fed mice. ISME J. 2017;11(7):1667-79.

13. Nataraj BH, Ali SA, Behare PV, Yadav H. Postbiotics-parabiotics: the new horizons in microbial biotherapy and functional foods. Microb Cell Fact. 2020;19(1):1-22.

14. Everard A, Belzer C, Geurts L, Ouwerkerk JP, Druart C, Bindels LB, et al. Cross-talk between Akkermansia muciniphila and intestinal epithelium controls diet-induced obesity. PNAS. 2013;110(22):9066-71.

15. Ashrafian F, Shahryari A, Behrouzi A, Moradi HR, Keshavarz Azizi Raftar S, Lari A, et al. Akkermansia muciniphila-derived extracellular vesicles as a mucosal delivery vector for amelioration of obesity in mice. Front Microbiol. 2019;10:2155.

16. Plovier H, Everard A, Druart C, Depommier C, Van Hul M, Geurts L, et al. A purified membrane protein from Akkermansia muciniphila or the pasteurized bacterium improves metabolism in obese and diabetic mice. Nature Med. 2017;23(1):107.

17. Depommier C, Van Hul M, Everard A, Delzenne NM, De Vos WM, Cani PD. Pasteurized Akkermansia muciniphila increases whole-body energy expenditure and fecal energy excretion in diet-induced obese mice. Gut Microbes. 2020. https://doi.org/10.1080/19490976.2020.1737307.

18. Ottman N, Reunanen J, Meijerink M, Pietilä TE, Kainulainen V, Klievink J, et al. Pili-like proteins of Akkermansia muciniphila modulate host immune responses and gut barrier function. PLoS ONE. 2017;12(3): e0173004.

19. Ashrafian F, Keshavarz Azizi Raftar S, Shahryari A, Behrouzi A, Yaghoubfar $\mathrm{R}$, Lari A, et al. Comparative effects of alive and pasteurized Akkermansia muciniphila on normal diet-fed mice. Sci Rep. 2021:11(1):1-13.

20. Keshavarz Azizi Raftar S, Ashrafian F, Yadegar A, Lari A, Moradi HR, Shahriary $\mathrm{A}$, et al. The protective effects of live and pasteurized Akkermansia muciniphila and its extracellular vesicles against HFD/CCl4-induced liver injury. Microbiol Spectr. 2021;9(2):e00484-21.

21. Kang C-S, Ban M, Choi E-J, Moon H-G, Jeon J-S, Kim D-K, et al. Extracellular vesicles derived from gut microbiota, especially Akkermansia muciniphila, protect the progression of dextran sulfate sodium-induced colitis. PLoS ONE. 2013;8(10):e76520.

22. Chelakkot C, Choi Y, Kim D-K, Park HT, Ghim J, Kwon Y, et al. Akkermansia muciniphila-derived extracellular vesicles influence gut permeability through the regulation of tight junctions. Exp Mol Med. 2018;50(2):e450.

23. Stentz R, Carvalho AL, Jones EJ, Carding SR. Fantastic voyage: the journey of intestinal microbiota-derived microvesicles through the body. Biochem Soc Trans. 2018;46(5):1021-7.

24. Behrouzi A, Ashrafian F, Mazaheri H, Lari A, Nouri M, Riazi Rad F, et al. The importance of interaction between MicroRNAs and gut microbiota in several pathways. Microb Pathog. 2020. https://doi.org/10.1016/j.micpa th.2020.104200.

25. Park S-S, Lee Y-J, Song S, Kim B, Kang H, Oh S, et al. Lactobacillus acidophilus NS1 attenuates diet-induced obesity and fatty liver. J Endocrinol. 2018;237(2):87-100.

26. Ji Y, Park S, Chung Y, Kim B, Park H, Huang E, et al. Amelioration of obesityrelated biomarkers by Lactobacillus sakei CJLS03 in a high-fat dietinduced obese murine model. Sci Rep. 2019:9(1):6821.

27. Depommier C, Everard A, Druart C, Plovier H, Van Hul M, Vieira-Silva S, et al. Supplementation with Akkermansia muciniphila in overweight and obese human volunteers: a proof-of-concept exploratory study. Nat Med. 2019:25(7):1096-103. 
28. Bischoff SC, Barbara G, Buurman W, Ockhuizen T, Schulzke J-D, Serino M, et al. Intestinal permeability - a new target for disease prevention and therapy. BMC Gastroenterol. 2014;14(1):189.

29. Tilg H, Zmora N, Adolph TE, Elinav E. The intestinal microbiota fuelling metabolic inflammation. Nat Rev Immunol. 2020;20(1):40-54.

30. Ghosh SS, Wang J, Yannie PJ, Ghosh S. Intestinal barrier dysfunction, LPS translocation, and disease development. J Endocr Soc. 2020;4(2): bvz039.

31. Shin N-R, Lee J-C, Lee H-Y, Kim M-S, Whon TW, Lee M-S, et al. An increase in the Akkermansia spp. population induced by metformin treatment improves glucose homeostasis in diet-induced obese mice. Gut. 2014;63(5):727-35.

32. Ashrafian F, Behrouzi A. Comparative study of effect of Akkermansia muciniphila and its extracellular vesicles on toll-like receptors and tight junction. Gastroenterol Hepatol Bed Bench. 2019;12(2):163.

33. Jacouton E, Mach N, Cadiou J, Lapaque N, Clément K, Doré J, et al. Lactobacillus rhamnosus cncmi-4317 modulates fiaf/angptl4 in intestinal epithelial cells and circulating level in mice. PloS ONE. 2015;10(10): e0138880.

34. Longo M, Zatterale F, Naderi J, Parrillo L, Formisano P, Raciti GA, et al. Adipose tissue dysfunction as determinant of obesity-associated metabolic complications. Int J Mol Sci. 2019;20(9):2358.

35. Huo Y, Lu X, Wang X, Wang X, Chen L, Guo H, et al. Bifidobacterium animalis subsp. lactis $A 6$ alleviates obesity associated with promoting mitochondrial biogenesis and function of adipose tissue in mice. Molecules. 2020;25(7):1490.

36. Lin $\mathrm{D}$, Chun $\mathrm{T}-\mathrm{H}$, Kang L. Adipose extracellular matrix remodelling in obesity and insulin resistance. Biochem Pharmacol. 2016;119:8-16.

37. Azzu V, Vacca M, Virtue S, Allison M, Vidal-Puig A. Adipose tissue-liver cross talk in the control of whole-body metabolism: implications in non-alcoholic fatty liver disease. Gastroenterology. 2020. https://doi. org/10.1053/j.gastro.2019.12.054.

38. Kobyliak N, Abenavoli L, Mykhalchyshyn G, Kononenko L, Boccuto L, Kyriienko D, et al. A multi-strain probiotic reduces the fatty liver index cytokines and aminotransferase levels in NAFLD patients: evidence from a randomized clinical trial. J Gastrointest Liver Dis. 2018. https:// doi.org/10.15403/jgld.2014.1121.271.kby.

39. Everard A, Plovier H, Rastelli M, Van Hul M, de Wouters DA, Geurts L, et al. Intestinal epithelial N-acylphosphatidylethanolamine phospholipase D links dietary fat to metabolic adaptations in obesity and steatosis. Nat Commun. 2019;10(1):1-17.

40. Wu W, Lv L, Shi D, Ye J, Fang D, Guo F, et al. Protective effect of Akkermansia muciniphila against immune-mediated liver injury in a mouse model. Front Microbiol. 2017;8:1804.

41. Zhang L, Zhang Z, Li Y, Liao S, Wu X, Chang Q, et al. Cholesterol induces lipoprotein lipase expression in a tree shrew (Tupaia belangeri chinensis) model of non-alcoholic fatty liver disease. Sci Rep. 2015;5(1):1-12.

42. Dijk W, Kersten S. Regulation of lipoprotein lipase by Angpt/4. Trends Endocrinol Metab. 2014;25(3):146-55.

43. Li J, Li L, Guo D, Li S, Zeng Y, Liu C, et al. Triglyceride metabolism and angiopoietin-like proteins in lipoprotein lipase regulation. Clin Chim Acta. 2020;503:19-34.

44. Lichtenstein L, Mattijssen F, de Wit NJ, Georgiadi A, Hooiveld GJ, van der Meer R, et al. Angpt/4 protects against severe proinflammatory effects of saturated fat by inhibiting fatty acid uptake into mesenteric lymph node macrophages. Cell Metab. 2010;12(6):580-92.

45. Park D-Y, Ahn Y-T, Park S-H, Huh C-S, Yoo S-R, Yu R, et al. Supplementation of Lactobacillus curvatus HY7601 and Lactobacillus plantarum KY1032 in diet-induced obese mice is associated with gut microbial changes and reduction in obesity. PloS ONE. 2013;8(3): e59470.

46. Kim S, Lee Y, Kim Y, Seo Y, Lee H, Ha J, et al. Akkermansia muciniphila prevents fatty liver disease, decreases serum triglycerides, and maintains gut homeostasis. Appl Environ Microbiol. 2020. https://doi.org/10. 1128/AEM.03004-19.

47. Hsieh F-C, Lan C-CE, Huang T-Y, Chen K-W, Chai C-Y, Chen W-T, et al. Heat-killed and live Lactobacillus reuteri GMNL-263 exhibit similar effects on improving metabolic functions in high-fat diet-induced obese rats. Food Funct. 2016;7(5):2374-88.

48. Raftar SKA, Abdollahiyan S, Azimirad M, Yadegar A, Vaziri F, Moshiri A, et al. The anti-fibrotic effects of heat-killed Akkermansia muciniphila MucT on liver fibrosis markers and activation of hepatic stellate cells. Probiotics Antimicrob Proteins. 2021. https://doi.org/10.1007/ s12602-020-09733-9.

49. Zhao L. The gut microbiota and obesity: from correlation to causality. Nat Rev Microbiol. 2013;11(9):639-47.

50. Tabasi M, Ashrafian F, Khezerloo JK, Eshghjoo S, Behrouzi A, Javadinia $\mathrm{SA}$, et al. Changes in gut microbiota and hormones after bariatric surgery: a bench-to-bedside review. Obes Surg. 2019;29(5):1663-74.

51. Turnbaugh PJ, Bäckhed F, Fulton L, Gordon Jl. Diet-induced obesity is linked to marked but reversible alterations in the mouse distal gut microbiome. Cell Host Microbe. 2008;3(4):213-23.

52. Turnbaugh PJ, Hamady M, Yatsunenko T, Cantarel BL, Duncan A, Ley $\mathrm{RE}$, et al. A core gut microbiome in obese and lean twins. Nature. 2009;457(7228):480-4.

53. Zhang X, Zhao Y, Zhang M, Pang X, Xu J, Kang C, et al. Structural changes of gut microbiota during berberine-mediated prevention of obesity and insulin resistance in high-fat diet-fed rats. PloS ONE. 2012;7(8): e42529.

54. Al K, Sarr O, Dunlop K, Gloor GB, Reid G, Burton J, et al. Impact of birth weight and postnatal diet on the gut microbiota of young adult guinea pigs. PeerJ. 2017;5: e2840.

55. Koliada A, Syzenko G, Moseiko V, Budovska L, Puchkov K, Perederiy $\checkmark$, et al. Association between body mass index and firmicutes/bacteroidetes ratio in an adult Ukrainian population. BMC Microbiol. 2017;17(1):120.

56. Ley RE, Turnbaugh PJ, Klein S, Gordon JI. Human gut microbes associated with obesity. Nature. 2006;444(7122):1022-3.

57. Magne F, Gotteland M, Gauthier L, Zazueta A, Pesoa S, Navarrete P, et al. The firmicutes/bacteroidetes ratio: a relevant marker of gut dysbiosis in obese patients? Nutrients. 2020;12(5):1474

58. Jiao X, Wang Y, Lin Y, Lang Y, Li E, Zhang X, et al. Blueberry polyphenols extract as a potential prebiotic with anti-obesity effects on C57BL/6 J mice by modulating the gut microbiota. J Nutr Biochem. 2019;64:88-100.

59. Liu Z, Chen Z, Guo H, He D, Zhao H, Wang Z, et al. The modulatory effect of infusions of green tea, oolong tea, and black tea on gut microbiota in high-fat-induced obese mice. Food Funct. 2016;7(12):4869-79.

60. Graham C, Mullen A, Whelan K. Obesity and the gastrointestinal microbiota: a review of associations and mechanisms. Nutr Rev. 2015;73(6):376-85.

61. Liang $Y$, Liang S, Zhang $Y$, Deng $Y$, He Y, Chen $Y$, et al. Oral administration of compound probiotics ameliorates HFD-induced gut microbe dysbiosis and chronic metabolic inflammation via the $\mathrm{G}$ proteincoupled receptor 43 in non-alcoholic fatty liver disease rats. Probiotics Antimicrob Proteins. 2019;11(1):175-85.

62. Thiennimitr P, Yasom S, Tunapong W, Chunchai T, Wanchai K, Pongchaidecha A, et al. Lactobacillus paracasei HII01, xylooligosaccharides, and synbiotics reduce gut disturbance in obese rats. Nutrition. 2018;54:40-7.

63. Cani PD, Delzenne NM. Gut microflora as a target for energy and metabolic homeostasis. Curr Opin Clin Nutr Metab Care. 2007;10(6):729-34.

64. Cani PD, Neyrinck AM, Fava F, Knauf C, Burcelin RG, Tuohy KM, et al. Selective increases of bifidobacteria in gut microflora improve highfat-diet-induced diabetes in mice through a mechanism associated with endotoxaemia. Diabetologia. 2007;50(11):2374-83.

65. Verdam FJ, Fuentes S, de Jonge C, Zoetendal EG, Erbil R, Greve JW, et al. Human intestinal microbiota composition is associated with local and systemic inflammation in obesity. Obesity. 2013;21(12):E607-15.

66. Zhu L, Sha L, Li K, Wang Z, Wang T, Li Y, et al. Dietary flaxseed oil rich in omega-3 suppresses severity of type 2 diabetes mellitus via antiinflammation and modulating gut microbiota in rats. Lipids Health Dis. 2020;19(1):1-16.

67. Turnbaugh PJ, Ley RE, Mahowald MA, Magrini V, Mardis ER, Gordon J. An obesity-associated gut microbiome with increased capacity for energy harvest. Nature. 2006:444(7122):1027-31.

68. Ley RE, Bäckhed F, Turnbaugh P, Lozupone CA, Knight RD, Gordon J. Obesity alters gut microbial ecology. PNAS. 2005;102(31):11070-5.

69. Zhang Y, Yu K, Chen H, Su Y, Zhu W. Caecal infusion of the short-chain fatty acid propionate affects the microbiota and expression of inflammatory cytokines in the colon in a fistula pig model. Microb Biotechnol. 2018;11(5):859-68. 
70. Johnson JL, Jones MB, Cobb BA. Polysaccharide A from the capsule of Bacteroides fragilis induces clonal CD4+ T cell expansion. J Chem Biol. 2015;290(8):5007-14.

71. Ding Y, Song Z, Li H, Chang L, Pan T, Gu X, et al. Honokiol ameliorates high-fat diet-induced obesity of different sexes mice by modulating the composition of the gut microbiota. Front Immunol. 2019;10:2800.

72. Kelder T, Stroeve J, Bijlsma S, Radonjic M, Roeselers G. Correlation network analysis reveals relationships between diet-induced changes in human gut microbiota and metabolic health. Nutr Diabetes. 2014;4(6):e122.

73. Derrien M, Vaughan EE, Plugge CM, de Vos WM. Akkermansia muciniphila gen. nov., sp. nov., a human intestinal mucin-degrading bacterium. Int J Syst Evol Microbiol. 2004;54(5):1469-76.

74. Leek JT JW, Parker HS, Fertig EJ, Jaffe AE, Storey JD, Zhang Y, Torres LC. sva: surrogate variable analysis. R package version 3.32.1. 2019.

75. Yang Y-W, Chen M-K, Yang B-Y, Huang X-J, Zhang X-R, He L-Q, et al. Use of $16 S$ rRNA gene-targeted group-specific primers for real-time PCR analysis of predominant bacteria in mouse feces. Appl Environ Microbiol. 2015;81(19):6749-56.

\section{Publisher's Note}

Springer Nature remains neutral with regard to jurisdictional claims in published maps and institutional affiliations.

- fast, convenient online submission

- thorough peer review by experienced researchers in your field

- rapid publication on acceptance

- support for research data, including large and complex data types

- gold Open Access which fosters wider collaboration and increased citations

- maximum visibility for your research: over $100 \mathrm{M}$ website views per year

At BMC, research is always in progress.

Learn more biomedcentral.com/submissions 